\title{
Shadow Detection by Local Color Constancy
}

\author{
Deepika Digarse \\ Sagar Institute of Research and Technology
}

\author{
Krishna Chauhan \\ Sagar Institute of Research and Technology
}

\begin{abstract}
This paper describe the technique of shadow detection properly, this technique can detect both the cast and selfshadow. The method exploits local color constancy properties which are cause of reflectance suppression in excess of shadowed regions. For detecting shadowed areas in a scene, the values of the backdrop image are separated by values of the current frame in the true color (RGB) space. We use all three type of colour space in our work. Illumination map is extracted using a steerable filter framework based on global, local correlations in low and high frequency bands respectively. The lighting and colour features so extracted are then input to a decision trees are designed to detect shadow edges using AdaBoost. The simulation results give us an idea about the performance of the proposed method as good with boundary marking on shadow and nonshadow region with high accuracy.
\end{abstract}

\section{Keywords}

Shadow detection, Amplitude Modulation \& Luminance Modulation, Colour Feature segmentation and Feature extraction, Illumination Map, Condition Random Field (CRF)

\section{INTRODUCTION}

Shadow is named as a region which created due obstruction of light source or rays Shadows in images are typically affected by a number of phenomena in any type of scene, which includes physical phenomena like; lighting conditions, manners of shadowed surfaces, objects, etc. The region, where the direct light source is completely blocked is called the umbra; on the other side the region, where it is partially blocked is known as the penumbra [1].At the time light sources are colorless and there is no color merging among objects, This type of shadow is often known as achromatic shadow, and those that are not achromatic is referred to as chromatic shadows. These techniques are portioned in the form of model-based and property-based techniques. Model-based techniques are explain by geometry of the scene, the illumination or the acquisition system.[1]. Our proposed method is a selective, loom on the basic of region which exploits the properties of the color constancy field, which comes in world over shadowed regions since of the effect of reflectance suppression. Local Color Constancy Detection method kind of color modal is used which compares intensity with respect to the chromaticity of each pixel. Lately data driven approaches are derived proposed for single image detection. This method learns to detect shadow based on training images. Zhu et al. classify region based on illumination intensity, texture and odd order derivatives for shadow region CRF (Condition Random Filed) optimization. CRF-based optimization is provides rational shadow contours. A shadow occurs when an object partially or totally occludes straight light from a light source, which is an ever-present aspect of our visual experience [1]. Shadows in images have long been troublemaking to computer vision problems, like tracking, recognition, object segmentation and many more. In practice, shadows cause problems such as shape alteration, thing merging and failure of object recognition and segmentation. Shadows are having double-face effect on scene understanding, depending on whether we model the shadows or ignore them. Generally, the region where a direct light source is entirely blocked is called the umbra, whereas the region where the region is incompletely blocked is known as the penumbra. Noticeably, both of them occur by a change of illumination. This illumination change is often measured only as a decline in brightness, without major change in chromaticity. However, the assumption only works when the light sources are white and there is no colour blending among objects. This category of shadow is habitually called an achromatic shadow, whereas those that are not achromatic are referred to as chromatic shadows [2]. For some conditions, a shadow is really a local change in both the colour and intensity of the scene illumination [3]. Estimating the colour of existing scene's lighting cause a problem which has taken much attention [4-7].Computing colour constancy is an illposed problem $[8,9]$. Often, these colour constancy algorithms are derived under some restrictive conditions or assumptions. Removing mono or di- color Shadows (chromatic) is also a particularly challenging task because of the fact that they are extremely difficult to distinguish from the foreground which has undefined pattern. Amato et al. [2] proposed to use the luminance ratio to identify segments with low gradient constancy, which in turn distinguish shadows from forefront. This algorithm achieved state of the art results on tricky video conditions. Huang and Chen [10] use a Gaussian mixture model (GMM) to learn colour features that are robust to the changes of surroundings surfaces or illuminant colour in a scene. Shadow region detection using the condition random field model Shadow regions and non-shadow regions differ with respect to illuminant intensity, texture, colour appearance. The rest of the paper is summarized as follows. The section II covers the outline of the algorithm; section III explains the colour segmentation and feature extraction. The section IV experimental result and the section V conclude the paper.

\section{OUTLINE OF ALGORITHM}

In this paper, intensity features from illumination maps and colour features from original images are combined to address the problem of shadow detection. The benefit of adding illumination features is that luminance changes due to changes in albedo will have already been removed from such maps. Thus this map offers robust features for detecting shadow regions. However, the approach of Lalonde et al. provide only extracted colour ratio and texture features from an image to use as inputs to a supervised learning method. The structure of our approach is captured in Figs 1 and 2. The upper lane corresponds broadly to the approach of Lalonde et al. without their texture features. At the stage trained the decision tree (Decision Tree 1) produces an initial shadow edge map. We use this to help improve the illumination map estimation process which is itself an improved version of the algorithm of Jiang et al., and is the lower path in Fig. 1. The illumination estimation process is exposed in Fig. 2. We give the impression of being up on Jiang et al.'s intrinsic image extraction framework [7] in order to obtain more accurate illumination maps in higher frequency bands. 


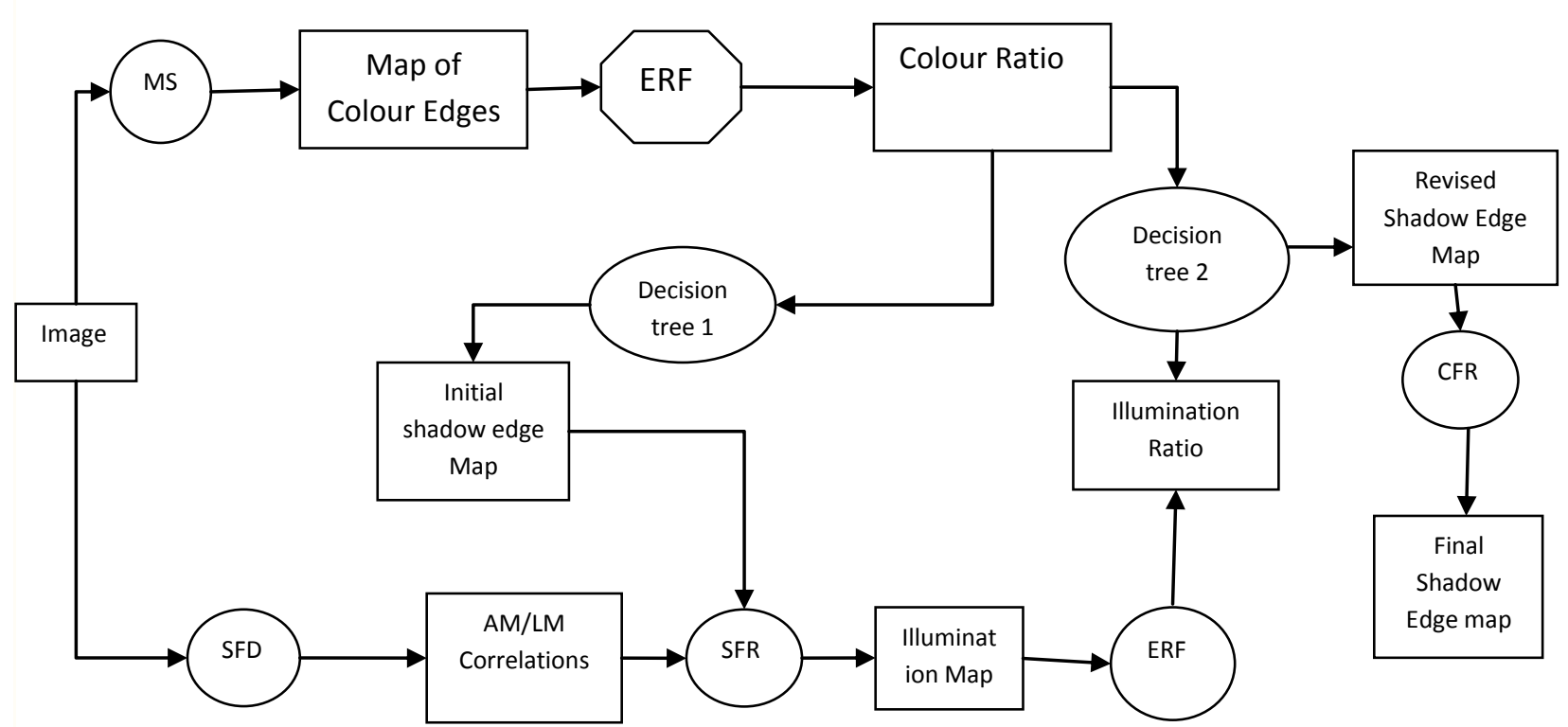

MS: Mean Shift Based Color Segmentation; ERF: Extract Ratio Feature; CRF: Condition Random Field; SFD: Steerable Filter Decomposition; $\quad$ SFR: Steerable Filter Reconstruction;

Figure 1: A flow chart giving a high level overview of our proposed algorithm.

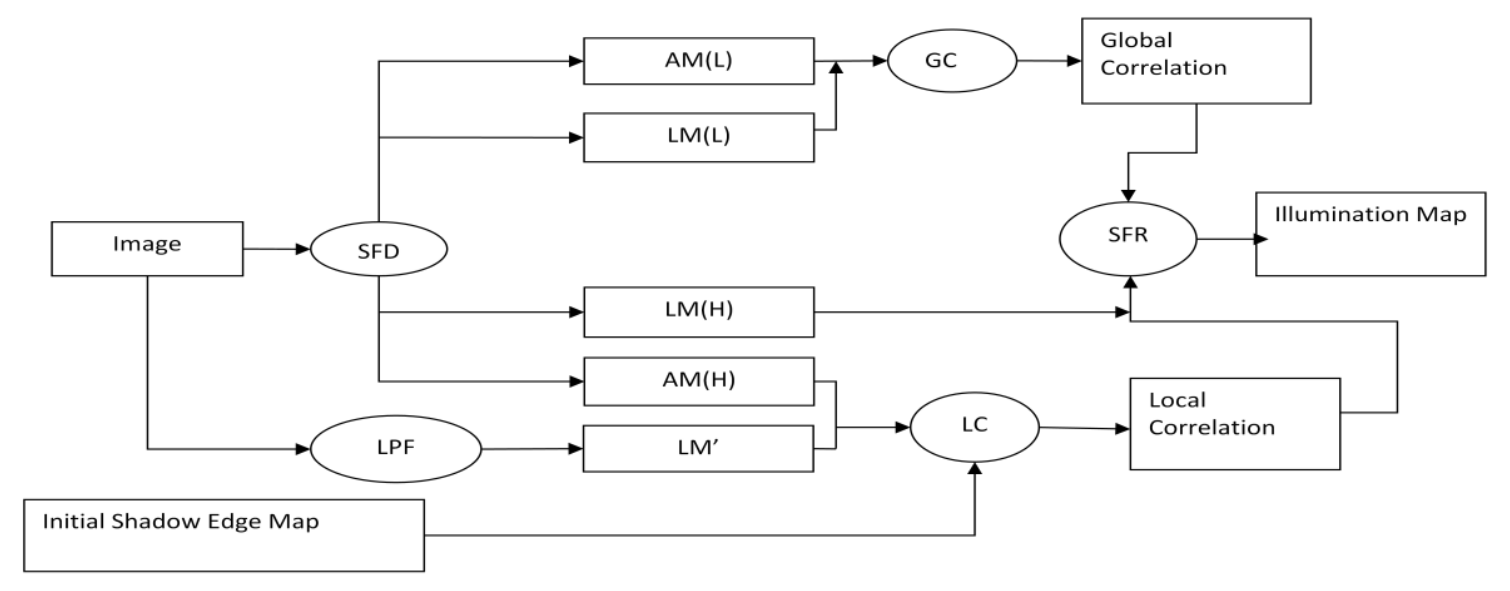

SFD: Steerable Filter Decomposition; LPF: Low Pass Filter; LC: Local Correlation

GC: Global Correlation; SFR: Steerable Filter Reconstruction

Figure 2: A flow chart giving detail on the improved approach for estimating the illumination map. This corresponds to the first two stages of the bottom row in Fig. 1. LM' is calculated directly from unrecompensed image. $H$ and $L$ refer to the outputs of steerable filters in High and Low frequency bands respectively. The SFD gives response up to 8 frequency and 8 orientation bands.

The Jiang et al. look for global correlations in between AM (Amplitude Modulation or local luminance contrast) and LM (Luminance Modulation or average local luminance) to note down divergence between the amount of reflectance changes (where AM and LM are uncorrelated); illumination changes (where AM and LM are correlated). This global strategy response is good in case for low frequencies, In case when apply this high frequency changes. We that's why estimate local correlations of LM and AM, and also individually between LM and colour features. Both these help us to separate high-frequency filter responses, at a local level, for later re-enactment into illumination or reflectance maps. For lower-frequency bands, global correlation in between luminance and contrast in each band still determines the corresponding weights for reconstruction [7]. Has an enhanced version of the separation of intrinsic images in lower alleyway, we then educe features from the illumination map. Using these illumination ratio features and the colour features extracted in the upper pathway, a second decision tree (also trained using AdaBoost) produces an improved estimate of the shadow edge probability map. This more effectively discriminates shadow edges from reflectance edges. This 
revised shadow edge probability map can then be improved further using a Conditional Random Field model with constraints that edges should be continuous and that neighboring pixels should have the same state.

\section{COLOUR SEGMENTATION AND FEATURE EXTRACTION}

We now describe the colour segmentation and feature extraction method. Colour features are used to assist the recognition for shadows straightly, and to get better value of illumination maps formed by the steerable filter method. We initially produce a colour segmentation using the mean shift algorithm [4] in (RGB) true color model. The edges of the same colour region will be labelled as shadow or non-shadow edges as shown later. Having indomitable the locations of colour edges we extract colour ratios (CR) across edges. The ratio is between the minimum and maximum values in different colour channels across a periphery [11]. Shadow edges are due to illumination changes so illumination ratio for regions either side of shadow boundaries should be the alike everywhere in the image, hence hue should be roughly stable across shadow boundaries. Non-shadow edges will not be so constrained. It is uncertain which colour space is the best to estimate these ratios, accordingly we estimate colour ratios in 3 kind of spaces (RGB, HSV and LAB) and at 4 different scales. In entire, come across out a 36-dimensional vector for every edge pixel in the image. Following Lalonde et al., we then use an AdaBoost-based decision tree, trained on a set of labeled images, to classify boundaries as shadow or nonshadow (see Section 5). This decision tree gives the probability that each edge pixel found by the mean shift algorithm is a shadow edge. The result of applying the decision tree is the initial shadow edge probability map which is used as input to the illumination map estimation process (by helping to separate reflectance and illumination changes, see Section 3). The colour features at current stage are also input openly into the final shadow detection process along with the illumination features. Intensity values in natural images or scene are the product of reflectance and illumination, and do not always represent the intrinsic features of the panorama, like the illumination profile, surface orientation/surface colors. It can be advantageous to separate illumination changes from reflectance changes: a type of intrinsic image extraction. Jiang et al. [7] estimated a kind of filter (steerable filter) based framework for calculating the values of the correlation between luminance and local contrast, colour texture at each frequency and orientation band, and it after it is followed by used the ensuing correlation coefficients to derive weights for each component of steerable filter output in order to reconstruct the illumination and reflectance maps. Because of its global nature, this method works well on low but not for high-frequency bands since the source and so appropriate categorization of high frequency components tends to vary athwart the image. Thus, we proposed to calculate local correlations in each band and apply these correlations to derive local rather than global weights. At the end of this section we show how if we calculate both local and global correlations we can determine the frequency band at which to shift from one to another, we refer for a hybrid correlation method.

\subsection{Local correlation between $L M$ and AM}

When illumination changes falls across a visual texture the luminance difference (amplitude, absolute contrast) between the light and dark parts of the texture also varies with the illumination. This is the positive correlation among illumination and local amplitude use to spot the shadow limits
[18]. Presume a images are being decomposes in the number of frequency and orientation bands $I_{i, j}(\mathrm{i}=1 ;:: ; \mathrm{N} ; \mathrm{j}=1$; $:: ; \mathrm{M})$ where $\mathrm{N}$ is the number of frequency bands and $\mathrm{M}$ the number of orientation bands. Modulations of local amplitude (AM) can be represented as the envelope of the high frequency components in Iij. Intended to u put in practice, we can be extract low frequency information from the amplitude of the high frequency components in the original image, i.e. $F_{l}$ (abs $\left.\left(I_{i, j}\right)\right)$, where $\mathrm{Fl}$ is a low-frequency filter. In the same way, local luminance modulation (LM) can be straight extracted via low-frequency filtering, i.e $F_{l}(I)$ If we have already segmented the image into regions based on colour (e.g. Fig.4(b)) we need only examine correlations between LM and AM in local regions on either side of colour edges. Let us indicate the set of colour perimeter pixels in the colour segmentation to be $\mathrm{D}=F_{l}^{d_{k}} ; d_{k} \ldots g$ so that $d_{k}$ indexes a point in any the original image $\mathrm{I}$, or any of the filter output $I_{i, j}$. Colour is consisting of both luminance and hue, and hence colour boundary pixels will be a superset of the shadow edge pixels. As a outcome we could do with only inspect the regions around these colour boundaries to find the shadow edges. If the correlation among LM and AM is strong, then at second step we ration the associated edge components for illumination map, or else for reflectance map. The local correlation - for a pixel $d_{k}$ - between AM and LM for the $i^{t h}$ frequency band and the $j^{t h}$ orientation band is denoted by $W_{i j}^{l}\left(d_{k}\right)$ and is defined as

$$
W_{i j}^{A M}\left(d_{k}\right)=\operatorname{cor}\left[F_{l}^{d_{k}}\left(\operatorname{abs}\left(I_{i j}\right)\right), I_{l}^{d_{k}}(I)\right]
$$

Where $W_{i j}^{A M}(P)=0$ for any pixel $\mathrm{p}=2 \mathrm{D}, \operatorname{cor}\left[s_{1} ; s_{2}\right]$ calculates correlation of two components $s_{1}$ and $s_{2}$, and $F_{l}^{d_{k}}(:)$ is the production of the filter something like edge pixel $\mathrm{dk}$. To analyze the local correlations we have to be cautious for selecting the suitable region in input image. Therefore if AM is calculated in a local window including these edge pixels the correlation with LM will compromised. In observation, the values of $\mathrm{LM}$ and $\mathrm{AM}$ in regions adjoining to every edge are the actual concern.

\subsection{Illumination Map Refining}

As described in Section 2, a decision tree based classifier is use for estimating the probability of a luminance edge being a shadow edge on the basis of colour features. We represent this probability as $W c(d k)$ for the boundary pixel dk. And now we have to bring into being a reconstructed illumination map based on the local correlation and it is the initial shadow edge map. The weights of each one component in this reconstruction are different for each edge pixel. We denote these weights $W_{i j}^{l}\left(d_{k}\right)$; where 1 refers to the fact that the reconstruction is partly based on the local correlation (as opposed to the global correlation) just put it is the addition of $W c(\mathrm{dk})$ and the local correlation $W_{i j}^{A M}\left(d_{k}\right)$ for each edge pixel $d k$,

$$
W_{i j}^{l}\left(d_{k}\right)=W^{c}\left(d_{k}\right)+W_{i j}^{A M}\left(d_{k}\right)
$$




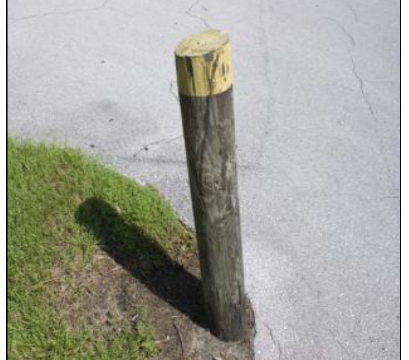

(a)

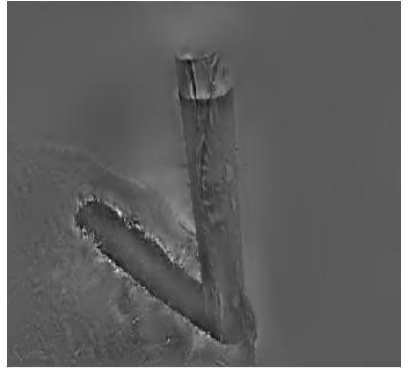

(c)

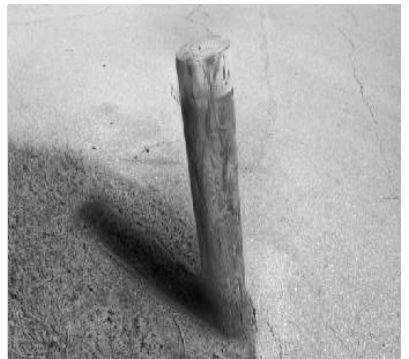

(e)

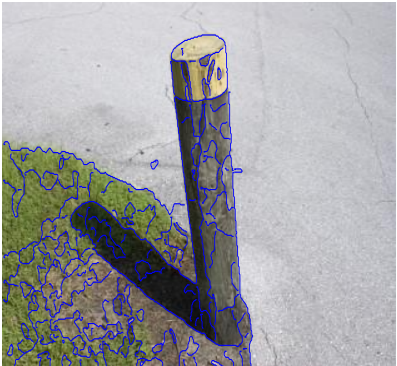

(b)

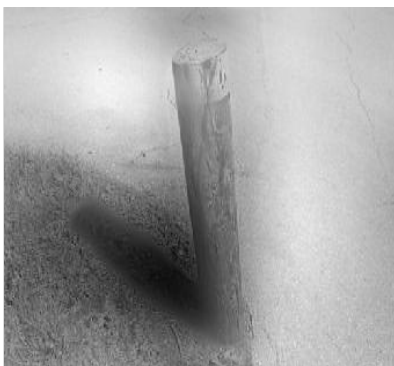

(d)

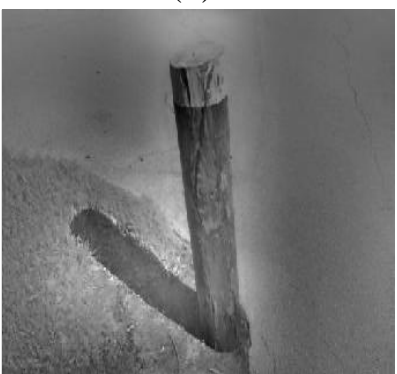

(f)
Figure 3: Intermediate results. (a) original image. (b) Segmentation result. (c) initial shadow edge map. (d) final shadow edge map. (e) illumination map (IM) reconstructed with global correlation. (f) IM from local correlation.

By instinct, the Decision Tree 1 based on colour features point toward that, for a shadow edge, we should deal out the edge to the illumination map instead of reflectance. $W_{i j}^{l}\left(d_{k}\right)=0$ for any pixel $\mathrm{p}=2 \mathrm{D}$.

\subsection{Hybrid correlation}

As the value of frequency is about to increase the steerable filter decomposes fine details of the image, and are dominated by local features. Consequently a local correlation policy is well again for the high frequency bands in comparison of the global strategy which is improved for lower frequency components. In our hybrid strategy we therefore merge the local and global strategies proceeding for reconstructing the reflectance and illumination maps. Reconstruction of the illumination map proceeds as follows:

$$
I M=\sum_{i=1}^{T-1} F_{i n v}\left(W_{i j}^{g} \times I_{i j}\right)+\sum_{i=T}^{N} F_{i n v}\left(W_{i j}^{l} \times I_{i j}\right)
$$

$W_{i j}^{g}$ Is the global correlation, and $\mathrm{T}$ is threshold frequency above which the local correlation strategy is applied (note: big $\mathrm{i}=$ high frequency filter). $\mathrm{T}$ is determined from the energy distribution of the global correlation measure $W_{i j}^{g}$ as follows,

$$
T=\arg _{t} \min \left(\sum_{i=1}^{t} \sum_{j=1}^{M} W_{i j}^{g} / \sum_{i=1}^{N} \sum_{j=1}^{M} W_{i j}^{g}>\lambda\right)
$$

Where $\lambda$ is representing the threshold energy. We set $1=0: 9$ in this paper. Fig.4 (e-g) shows exemplar reconstructed illumination maps using global, local and hybrid correlations.

The hybrid method characterizes the shadow best, retaining its edges and intensity.

\subsection{Shadow detection}

We now combine the colour features described in Section 2 and illumination information into a new shadow edge classifier trained on features from both of sources. The first step of our work is to estimate intensity ratios in the illumination map from regions on either side of colour boundaries. As with colour ratios we compute the intensity ratios at 4 dissimilar scales. Taken with the colour features, it gives a 40-dimensional feature vector for every boundary pixel recognized by the mean shift method. We skilled a new decision tree (Decision tree 2 in Fig 1) for classifying pixels as shadow or non-shadow. The decision tree gives the probability value pshd of each pixel being on a shadow boundary. To further refine the resulting classifications, we introduce conditional random field (CRF) model $[9,21]$ built with the constraint that edges should be continuous. The full cost function can be written as:

$$
E=\lambda \sum_{i} F_{d}\left(P_{i}, l_{P i}\right)+\sum_{j(j \neq i)} F_{S}\left(l_{P i}, l_{P j}\right)
$$

where $\lambda$ the data cost is $F_{d}\left(P_{i} ; l P_{i}\right)$; the smoothing cost is $F_{S}\left(l P_{i} ; l P_{j}\right)$; and 1 controls the balance between these two items. For the data cost, the penalty for labeling a pixel shadow or non shadow will be $-\log _{p}$ shd $-\log _{p}$ shd In order to reduce the influence of this prior, a regularization term is added, as a result that $F_{d}\left(P_{i} ; l P_{i}\right)$; is written as

$$
\left\{\begin{array}{c}
F_{d}\left(P_{i}, l_{P i}\right)= \\
-\log \left(P_{\text {shd }}\right)-\log \left(P_{C}\right) \text { if } l_{P i}=1(\text { shadow }) \\
-\log \left(1-P_{\text {shd }}\right)-\log \left(1-P_{C}\right) \text { if } l_{P i}=0(\text { non }- \text { shadow })
\end{array}\right.
$$

The constraint function, $F s(l P i$; $l P j)$ evaluates; the cost of giving label $l_{P i}$ to point $\mathrm{Pi}$, and label $l_{P i}$ to point $P j$, where $P i$ and $P j$ are adjacent to each other. If they belong to the identical colour edging, and have similar features, then they should have the same label. Otherwise, setting same labels should be penalized. Function Fs is defined as

$$
F_{S}=1\left(P_{i} \neq P_{j}\right) \exp \left(-\beta\left\|f_{P_{i}}-f_{P_{i}}\right\|_{2}^{2}\right)
$$

Where $1(:)$ is the indicator function; $f_{P i}$ is the colour ratio feature in HSV and LAB space of points Pi. The optimal label can be found through minimizing the whole cost function i.e. min E by means of the graph cut algorithm [9, 21].

\section{EXPERIMENTAL RESULTS}

Maximum of the algorithms tried were evaluated against the labeled dataset from which comprised mainly outdoor images including natural views, street views and satellite resultant images. Some shadow is comes into the view due to natural objects, like plants, mountains and stones, and some by manmade objects, such as buildings, cars, and lamp posts. We therefore tested our algorithm on the decisive factor, and on the supplementary difficult problem of general shadow edge detection. The Colour and illumination features balance each 
other, and as a result edge pixels are classified as shadows at the one condition hue is pretty constant across the edge and the change in illumination is consistent with the difference between sun and ambient lighting. Finally, adding the CRF model increased classification performance to $83: 5 \%$ correct rates. In this work we in step a false alarm rate by varying the regularization term $\mathrm{pc}$ in the $\mathrm{CRF}$. The other parameters were selected as $1=0: 5 ; b=4$ through cross-validation. In Comparison to shadow detection results based on colour features, the anticipated algorithm can remove the false alarms and amplify the possibility of true positives as well. The detection performance visually shown in figure 4 and comparatively in figure 5 the

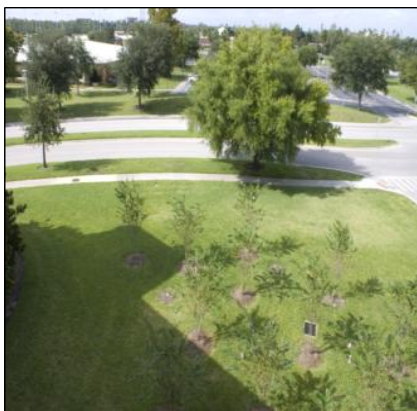

(a)

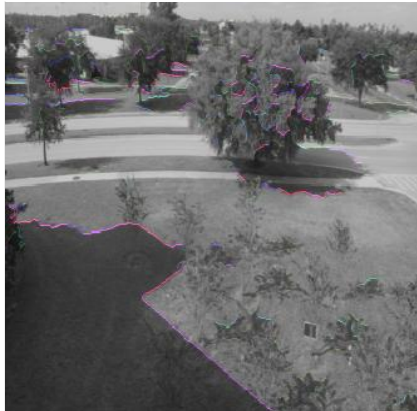

(c)

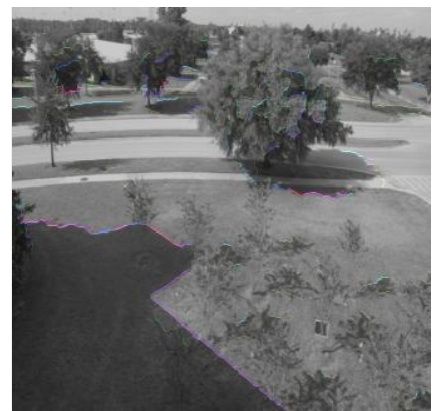

(b)

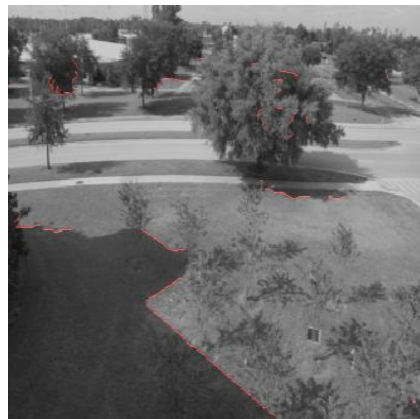

(d)
Figure 4: Shadow detection results. (a) Original Image (b) colour based shadow detection (c) Shadow detection based on colour and illumination. (c) shadow detection based on CFR.

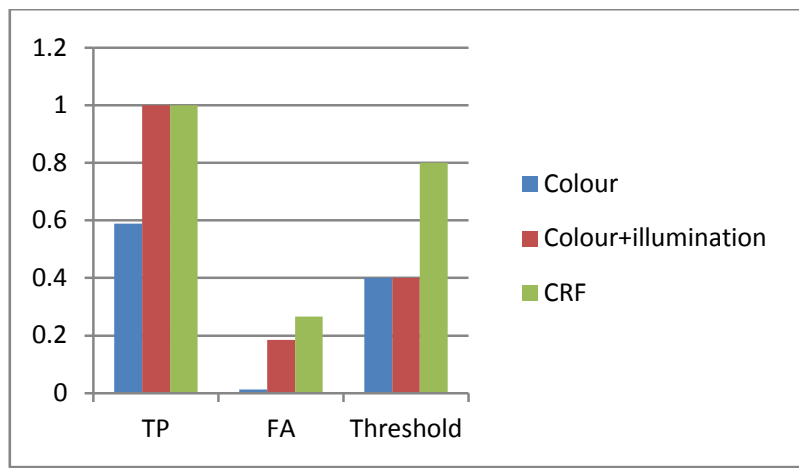

Fig.5 Graph for ground image

\section{CONCLUSION}

In the projected shadow recognition algorithm, features from illumination map, colour segmentation domino effect is combined as inputs to a decision tree trained to detect shadow limits (edges). A hybrid correlation strategy is proposed to for selecting filter responses for reconstructing the illumination maps. The proposed algorithm is moderately exact, outperforming one more kind of current methods. It is setting up to use shadow detection results to further refine intrinsic image taking out methods in an iterative fashion.

\section{REFERENCES}

[1] H.G. Barrow and J.M. Tanenbaum. Recovering intrinsic scene characteristics from images. CVS, pages 3-26, 1978.

[2] Bousseau, S. Paris, and F. Durand. User assisted intrinsic images. ACM Transactions on Graphics (Proceedings of SIGGRAPH Asia 2009), 28(5):130:1-130:10, 2009.

[3] M. Collins, Robert E. Schapire, and Y. Singer. Logistic regression, adaboost and bregman distances. Machine Learning, 48:253-285, September 2002.

[4] D. Comanicu and P. Meer. Mean shift: A robust approach toward feature space analysis. IEEE Trans. Pattern Analysis and Machine Intelligence, 24:603-619, 2004.

[5] G. D. Finlayson, S. D. Hordley, C. Lu, and M. Drew. On the removal of shadows from images. IEEE Trans. Pattern Analysis and Machine Intelligence, 28:59-68, 2006.

[6] Huerta, M. Holte, T. Moeslund, and J. Gonzalez. Detection and removal of chromatic moving shadows in surveillance scenarios. IEEE Int'l Conf. on Computer Vision, 2009.

[7] X. Jiang, A. J. Schofield, and J. L. Wyatt. Correlationbased intrinsic image extraction from a single image. ECCV, 4:58-71, 2010.

[8] J. Joshi and N. P. Papanikolopoulos. Learning to detect moving shadows in dynamic environments. IEEE Transactions on Pattern Analysis and Machine Intelligence, 30:2055-2063, November 2008.

[9] V. Kolmogorov and R. Zabih. What energy functions can be minimized via graph cuts. IEEE Transactions on Pattern Analysis and Machine Intelligence, 26: 65-81, 2004.

[10] J. F. Lalonde, A. A. Efros, and S.G. Narasimhan. Estimating natural illumination from a single outdoor image. IEEE Int'l Conf. on Computer Vision, pages 183 190, 2009.

[11] J. F. Lalonde, A. A. Efros, and S. G. Narasimhan. Detecting ground shadow in outdoor consumer photographs. ECCV, pages 322-335, 2010.

[12] N. M. Brisson and A. Zaccarin. Learning and removing cast shadows through a multi distribution approach. IEEE Transactions on Pattern Analysis and Machine Intelligence, 29:1133-1146, July 2007.

[13] Y. Matsushita, S. Lin, S. B. Kang, and H.-Y. Shum. Estimating intrinsic images from image sequences with biased illumination. ECCV, 2: 274-286, 2004.

[14] Y. Matsushita, K. Nishino, K. Ikeuchi, and M. Sakauchi. Illumination normalization with time-dependent intrinsic images for video surveillance. IEEE Transactions on Pattern Analysis and Machine Intelligence, 26:1336 1347, 2004

[15] T. Okabe, I. Sato, and Y. Sato. Attached shadow coding: Estimating surface normals from shadows under unknown reflectance and lighting conditions. In IEEE Int'1 ICCV, pages 1693-1700, 2009. 
[16] B. C. Russell, A. Torralba, K. P. Murphy, and W. T. Freeman. Labelme: A database and web-based tool for image annotation. International Journal of Computer Vision, 77:157-173, 2008.

[17] Sato, Y. Sato, and K. Ikeuchi. Illumination from shadows. IEEE Transactions on Pattern Analysis and Machine Intelligence, 25(3):290-300, 2003.

[18] J. Schofield, G. Hesse, P. B. Rock, and M. A. Georgeson. Local luminance amplitude modulates the interpretation of shape-from-shading in textured surfaces. Vision Research, 46:3462-3482, 2006.

[19] L. Shen, P. Tan, and S. Lin. Intrinsic image decomposition with non-local texture cues. IEEE CVPR, pages 1-7, 2008.

[20] E. P. Simoncelli and W. T. Freeman. The steerable pyramid: A flexible architecture for multi-scale derivative computation. IEEE Second Int'l Conf on Image Processing, pages 444-447, 1995.
[21] R. Szeliski, R. Zabih, D. Scharstein, O. Veksler, A. Agarwala, and C. Rother. A comparative study of energy minimization methods for markov random fields with smoothness-based priors. IEEE Trans. Pattern Analysis and Machine Intelligence, 30(6):1068-1080, 2008.

[22] M. F. Tappen, W. T. Freeman, and E. H. Adelson. Recovering intrinsic images from a single image. IEEE Trans. Pattern Anal. Mach. Intell., 27:1459-1472, 2005.

[23] E. Vazquez, R. Baldrich, J. V. d. Weijer, and M. Vanrell. Describing reflectances for color segmentation robust to shadows, highlights, and textures. IEEE Trans. Pattern Analysis and Machine Intelligence, 33:917-930, 2011.

[24] Y. Weiss. Deriving intrinsic images from image sequences. IEEE Int'l Conf. on Computer Vision, 2:6875, 2001.

[25] J. Zhu, K. G. G. Samuel, S. Z. Masood, and M. F. Tappen. Learning to recognize shadow in monochromatic natural images. CVPR 10: Proceedings of the 2006 IEEE CSCCVPR, pages 223-230, 2010. 